\title{
Standardized Precipitation Index (SPI) for Drought Intensity Assessment in South Kashmir
}

\author{
Yogesh Pandey ${ }^{1}$, Ram Nath Jha ${ }^{2 *}$, Sabah Parvaze ${ }^{1}$, Latief Ahmad $^{1}$ and Shahzad Faisal ${ }^{1}$ \\ ${ }^{1}$ College of Agricultural Engineering and Technology, SKUAST Kashmir, India \\ ${ }^{2}$ Agricultural Machinery Testing and Research Centre, Nawalpur, Sarlahi, Nepal \\ *Corresponding author
}

\section{A B S T R A C T}

\section{Keywords}

Drought, Drought

Monitoring,

Standardized

Precipitation Index,

Temporal pattern

\section{Article Info}

Accepted:

20 November 2019

Available Online:

10 December 2019
The present study deals with the potential of using precipitation-based Standardized Precipitation Index to analyse the temporal pattern of drought in South Kashmir area of Jammu and Kashmir. Monthly precipitation data from 1980 to 2017 for Anantnag region were used to compute Standardized Precipitation Index (SPI) values. The computation of SPI series was done for short, intermediate as well as for long time scales. Analysis of SPI values were done to study the temporal patterns of drought occurrence. The drought severity and duration were also estimated. From the analysis, droughts in the Anantnag region occurred during the years 1999, 2000, 2001 and 2016. The intensity of these droughts was found to be severe to extreme. The most prolonged droughts in the region occurred during three successive years during 1999, 2000 and 2001. The SPI values were less than -1 for these years on 6-month, 9-month and12-month time scales. The 12-month SPI values for these years were $-1.53,-1.56$ and -1.87 respectively. However, the SPI values suggest moderate dryness in place of acute dryness during the years of severe and extreme drought.

\section{Introduction}

Droughts are known as an environmental adversity and have enticed the attention of hydrologists, ecologists, environmentalists, geologists, meteorologists and agricultural scientists. Drought has different meanings for people belonging to different disciplines. A drought for an agriculturist may not to be so for a meteorologist or hydrologist. Thus, the definition of drought is different for different disciplines.
Droughts are known to occur in almost all climatic zones of the world. Droughts are typically related to the decrease in the quantity of precipitation received over a prolonged period of time, for instance a season or a year. Temperatures; high wind velocities; low relative humidity; timing and characteristics of rainfall, including distribution of rainy days during crop growing seasons, intensity and duration of rain, and onset and termination, play a important role in the occurrence of droughts. 
Drought is a state in which there is a deficiency of adequate moisture in the environment, essentially due to the dearth of precipitation or deficiency in precipitation in an area over a protracted period of time, usually a season or more, subsequent in a water scarcity causing unfavourable effects on vegetation, animals, and people. It is broadly defined as severe water shortage caused by low rainfall and drop in agricultural production. Although there is no universal definition of drought, this is the definition which many experts seem to agree on. Drought is different than aridity, which is an everlasting feature of climate in areas where low precipitation is the norm, as in a desert.

One of the most widely used indicator of drought is the deviation of rainfall from mean. This method is very simple but has a serious limitation of dependence on mean. Deviation of rainfall is not useful for drought monitoring in areas showing variation in mean rainfall. An area having high rainfall and another having low rainfall area can have the same rainfall deviation for two different amounts of actual rainfall. Therefore, rainfall deviations across space and time need to be interpreted with due care.

Standardized Precipitation Index (SPI) is an indicator of actual precipitation as a standardized departure with respect to rainfall probability distribution function. Thus, SPI has received an additional importance in recent years as a potential drought indicator allowing spatial and temporal comparisons.

The calculation of SPI needs long term data on precipitation to determine the probability distribution function (PDF) which is then transformed to a normal distribution. A normal distribution has mean zero and standard deviation of one. Thus, the values of SPI are stated in standard deviations. Positive values of SPI indicate higher than median precipitation and negative values indicate lower than median precipitation (Edwards and McKee, 1997). Since SPI values fit a typical normal distribution, the values lie in one standard deviation $(\sigma)$ nearly $68 \%$ of time, within $2 \sigma 95 \%$ of time and within $3 \sigma 98 \%$ of time (Wu et al., 2006). In recent past SPI has been used increasingly for assessment of drought intensity in many countries (VicenteSerrano et al., 2004; Vijendra et al., 2005; Giddings et al., 2005).

The drought interpretation at different time scales using SPI has been proved to be superior to Palmer Drought Index (Guttman, 1998; Goodrich and Ellis, 2006). The SPI (McKee et al. 1993, 1995) is a powerful, flexible index that is simple to calculate. In fact, precipitation is the only input parameter. Also, it is just as effective in analysing wet periods/cycles as it is in analysing dry periods/cycles.

For classification of the SPI drought severity, it is believed that the drought appears within a period when SPI is negative consistently, and drought starts when SPI is down to zero, and it stops when the severity values are positive (Mckee et al. 1993).

The present study analyses the response of various SPI values to drought situation vis-àvis comparison of SPI with actual rainfall and rainfall deviation from normal in Anantnag, South Kashmir. SPI has also been identified as an indicator of soil moisture stress. A strong correlation exists between 12-month SPI and soil moisture departure in the Kashmir valley (Ahmad et al. 2016). South Kashmir has a vast agricultural sector producing major crops of the valley like rice, maize and wheat. Thus, any drought in this area will affect the substantial farmer as well as the population. But no substantial studies on drought analysis have been performed for the region. Consequently, there arises a need to study 
pattern \& severity of drought in this region as drought are not possible to avoid but drought preparedness can be developed and drought impact can be managed.

\section{Study area}

Anantnag is one of the districts of Jammu and Kashmir with a total area of $3574 \mathrm{~km}^{2}(1380$ sq. mi) and is present at an elevation of $1600 \mathrm{~m}$ (5300ft). It lies between the geographical coordinates of $33^{\circ} 43^{\prime} 47^{\prime \prime} \mathrm{N}$ latitude and $75^{\circ}$ 8' 59" E longitude. Monthly precipitation data was obtained for three stations viz. Qazigund, Pahalgam, Kokernag from the India Meteorological Department. Data of these stations was obtained for a period of 38 years from 1980-2017.

Anantnag accentuates a moderate climate which is largely defined by its geographical location with Karakorum in the east and Pir Panjal range to the south. The district receives an average annual precipitation of $747 \mathrm{~mm}$ and has generally high relative humidity in summers where nights are cold. The average annual temperature for the district is recorded to be $14.5^{\circ} \mathrm{C}$. The study area and location of meteorological department is shown in the Figure 1.

\section{Materials and Methods}

The monthly precipitation data from the three meteorological stations was used to compute the average monthly precipitation at the three stations. The average precipitation of the region was then computed using Thiessens polygon method, which was then used to calculate SPI. McKee et al. framed the Standardized Precipitation Index (SPI) with the intention of monitoring drought. The departure of annual rainfall from the mean during the period 1980-2017 was also calculated. It is one of the simplest rain measurements for a location. It is calculated by using the following equation.

Departure $(\%)=\frac{\text { Actual Precipitation }- \text { Normal Precipitation }}{\text { Actual Precipitation }} \times 100$

The percentage departure from normal is a very simple index and makes presents a realistic method of communicating drought to the public.

For calculation of SPI, the long-term record of precipitation values is fitted to a probability distribution. The distribution is then transformed into a standard normal distribution. The mean of this standard distribution is 0 while the variance is 1 (Edwards and McKee, 1997). The datasets are most commonly adjusted to the gamma function. The statistics for the frequency distribution are figured on the basis of a reference time-scale of minimum 30 years. The SPI output values are in units of standard deviation from long-term medians and offer the corresponding probabilities of occurrence of each drought category relative to the normal probability density function. McKee et al. (Mc Kee et al., 1993) also set up a criterion for classification of drought conditions based on the SPI (Table 1).

The intensity of drought intensity is defined by the value of SPI. In general, SPI values greater than zero represent greater than normal precipitation, while SPI values less than zero represent negative values indicate lower than normal precipitation. The SPI can be computed for different time scales and can provide early warning of drought, although it is not a forecasting tool. The duration of drought event/spell may be obtained by counting the months from the beginning to ending of negative SPI values and magnitude by positive summing the SPI values of all months within drought event/spell. In this study, SPI was computed at timescales of 1month, 3-months, 6-months, 9-months and 12months. 


\section{Results and Discussion}

The analysis of the present study was focused on agreement of the sensitivity of SPI to actual rainfall departures and the behaviour of SPI in drought and normal years. The SPI based drought classes proposed by Mc Kee et al. (1993), have been implemented in this study. The method has found its applicability to different climatological regions of the world (Giddings, 2005; Loukas et al., 2004; Morid et al., 2006; Bartolini et al., 2008; Paulo et al., 2005). SPI has also been used for the drought quantification of Jhelum River Basin in Kashmir valley (Parvaze et al. 2018).

\section{Departure analysis}

Annual Rainfall Departure For identification of drought years and the extent of deficit of annual rainfall, the annual rainfall departure analysis has been carried out. A year is considered as drought year if the total amount of annual rainfall over an area is deficient by more than $25 \%$ of its normal value. The percentage annual rainfall departures in all blocks of Anantnag district are given in Figure. From the analysis it is observed that in years 2002,2016 and 2017 as moderate drought and throughout these 38 years no severe drought occurred in Anantnag district, the chance of occurrence of drought in every 10 years varies from 0.1 to 1 . It means that year after every 9 to 10 year is a drought year.

Also, trend analysis of precipitation showed a slight decrease of precipitation in the last 30 years for the region with linear regression equations $\mathrm{y}=-0.4141 \mathrm{x}+6.6137$ and $\mathrm{R}^{2}=$ 0.0225

\section{Analysis of SPI on different timescales}

The annual SPI for Anantnag from 1980 to 2017 was calculated to demonstrate the frequency of occurrence of dry and wet conditions. The SPI based drought classification proposed by McKee et al. (1993) was adopted in this study. The value of the SPI gives a measure of the severity of a wet or dry event. A drought event occurs if the SPI value is -1.0 or less and the event ends when the index becomes positive (Bordi et al. 2001). SPI was computed for various timescales. The temporal behaviour of the SPI values is presented in graphs below. It was observed that on smaller scales such as SPI-1 and SPI-3 series, the drought intensities are highly variable and become less than -1.0 and greater than 1.0 on several occasions. However, on longer timescales such as SPI-12 drought intensity decreases. This variation is due to a seasonal component found in the rainfall data since SPI is relative to the rainfall characteristics of that area.

SPI calculations showed that the lowest SPI on the 1-month timescale for Anantnag district was -3.15 which occurred in the year 1990. On 3-month timescale, the lowest SPI for Anantnag district was -3.01 which occurred in the year 2016. The lowest SPI on 6-month timescale is -2.45 during the year 2001. Also, the lowest SPI on 9-month time scale was 2.45 which occurred during the year, 2000. Moreover, the lowest SPI on 12-month timescale was -2.42 for Anantnag district in the year 2016 .

For short (1-month) and intermediate (3month) timescales, the high temporal frequency was observed whereas for longer (12-month) timescales the frequency of occurrence of drought decreases.

\section{1-month SPI}

1-month SPI indicates short term conditions and it can be closely associated to the meteorological drought. It is useful for assessing soil moisture and crop stress, particularly during the growth season of the 
crops. The lowest SPI on 1-month time scale for the Anantnag was -3.15 which occurred the year of 1990 (Fig.3).

1-month SPI indicates short term conditions and it can be closely associated to the meteorological drought. It is useful for assessing soil moisture and crop stress, particularly during the growth season of the crops. The lowest SPI on 1-month time scale for the Anantnag was -3.15 which occurred the year of 1990 .

\section{3-Month SPI}

3-month SPI specifies short term as well as medium- term moisture conditions and offers an estimate of seasonal precipitation. 3-month SPI is more helpful in emphasizing existing moisture conditions of major agricultural regions than many other hydrological indices. On 3-month timescale, the lowest SPI for Anantnag district was -3.01 which occurred in the year 2016 (Fig. 4).

\section{6-Month SPI}

The 6-month SPI specifies seasonal to medium term trends in precipitation and is very efficient in showing the precipitation over different seasons. SPI calculations showed that the lowest SPI on 6-month time scale for Anantnag district is -2.45 during the year 2001(Fig.5).

\section{9-Month SPI}

The 9-month SPI provides an insight of interseasonal precipitation patterns over a time scale of medium intervals. For droughts to develop, generally a season or more is required. For a 9-month time scale, SPI value of -1.5 or below indicates that the dryness has a significant impact on agriculture as well as on other sectors. The lowest SPI on 9-month timescale for Anantnag district was -2.45 which occurred during the year of 2000 (Table 2).

Table.1 Drought categories from SPI (Mc Kee et al., 1993)

\begin{tabular}{|l|l|}
\hline SPI Values & Drought Category \\
\hline $2.00+$ & extremely wet \\
\hline $\mathbf{1 . 5 0}$ to 1.99 & very wet \\
\hline $\mathbf{1 . 0 0}$ to 1.49 & moderately wet \\
\hline$-\mathbf{0 . 9 9}$ to 0.99 & near normal \\
\hline $\mathbf{- 1 . 0 0}$ to -1.49 & moderate drought \\
\hline $\mathbf{- 1 . 5 0}$ to -1.99 & severe drought \\
\hline $\mathbf{- 2 . 0 0}$ and less & extreme drought \\
\hline
\end{tabular}


Table.2 Correlation between IMD criteria OF DROUGHT and SPI

\begin{tabular}{|c|c|c|}
\hline Year & $\%$ deviation from normal & 12-month SPI \\
\hline 1980 & -4.67 & -0.18 \\
\hline 1981 & 14.31 & 0.62 \\
\hline 1982 & 19.32 & 0.81 \\
\hline 1983 & 10.81 & 0.48 \\
\hline 1984 & -11.08 & -0.48 \\
\hline 1985 & -7.82 & -0.33 \\
\hline 1986 & 16.53 & 0.7 \\
\hline 1987 & 1.77 & 0.1 \\
\hline 1988 & 19.36 & 0.81 \\
\hline 1989 & -2.86 & -0.1 \\
\hline 1990 & -7.51 & -0.31 \\
\hline 1991 & -26.3 & -1.24 \\
\hline 1992 & 28.12 & 1.15 \\
\hline 1993 & 8.56 & 0.38 \\
\hline 1994 & 44.79 & 1.74 \\
\hline 1995 & 24.81 & 1.02 \\
\hline 1996 & 57.08 & 2.14 \\
\hline 1997 & 28.2 & 1.15 \\
\hline 1998 & -6.41 & -0.26 \\
\hline 1999 & -31.6 & -1.53 \\
\hline 2000 & -32.19 & -1.56 \\
\hline 2001 & -37.36 & -1.87 \\
\hline 2002 & -29.96 & -1.44 \\
\hline 2003 & 9.08 & 0.4 \\
\hline 2004 & -16.17 & -0.72 \\
\hline 2005 & 6.3 & 0.29 \\
\hline 2006 & 17.25 & 0.73 \\
\hline 2007 & -26.69 & -0.65 \\
\hline 2008 & -10.4 & -0.45 \\
\hline 2009 & -17.47 & -0.79 \\
\hline 2010 & 0.64 & 0.11 \\
\hline 2011 & -1.57 & 0.01 \\
\hline 2012 & -16.1 & -0.63 \\
\hline 2013 & -26.92 & -1.16 \\
\hline 2014 & 36.41 & 1.44 \\
\hline 2015 & 19.57 & 0.85 \\
\hline 2016 & -48.76 & -2.24 \\
\hline \multirow[t]{2}{*}{2017} & -1.1 & 0.03 \\
\hline & $\begin{array}{c}\text { CORRELATION } \\
\text { COEFFICIENT }(\mathbf{r})=\end{array}$ & 0.99 \\
\hline
\end{tabular}


Fig.1 Map of Anantnag district of Jammu and Kashmir

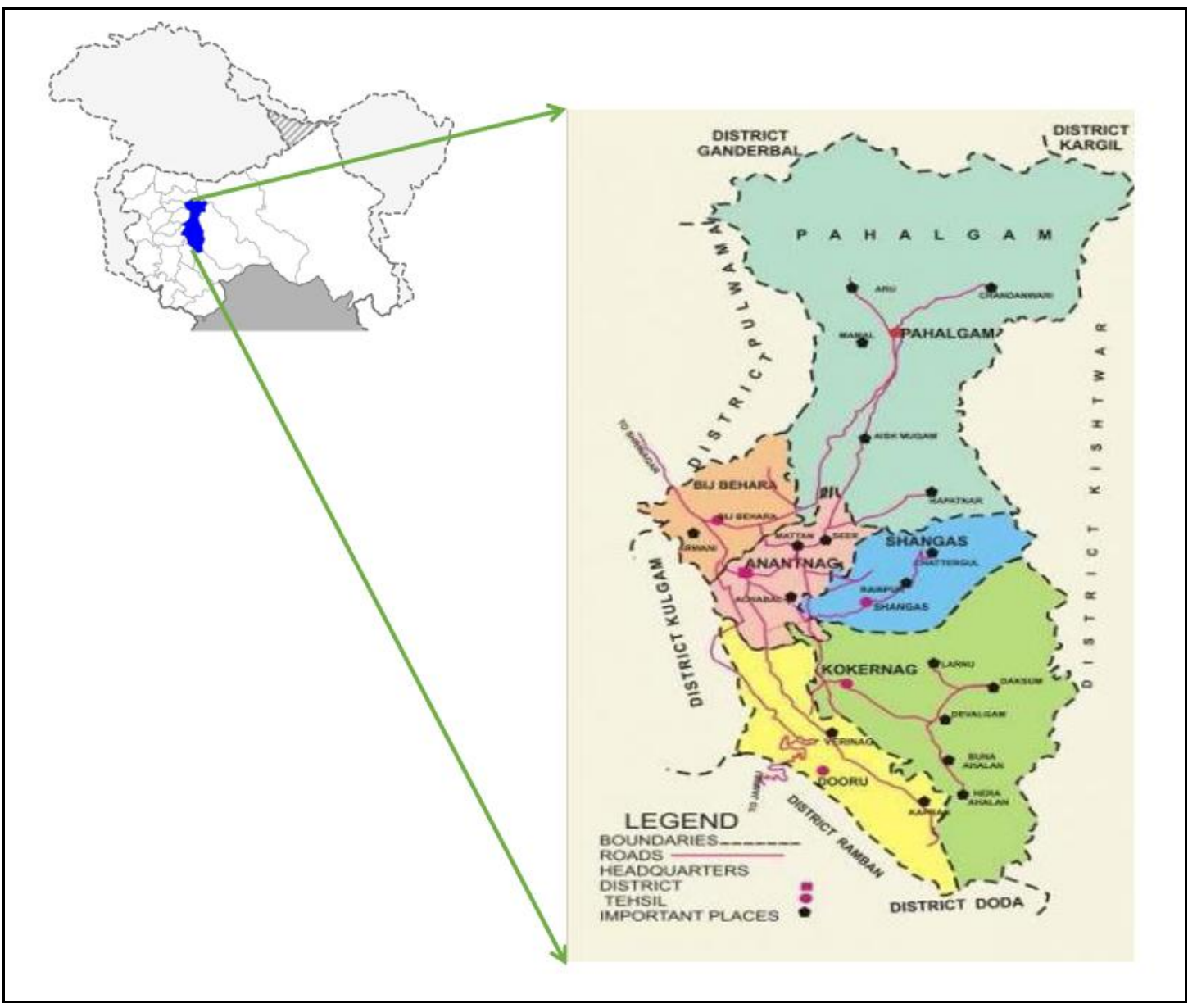

Fig.2 Departure Analysis of Anantnag District

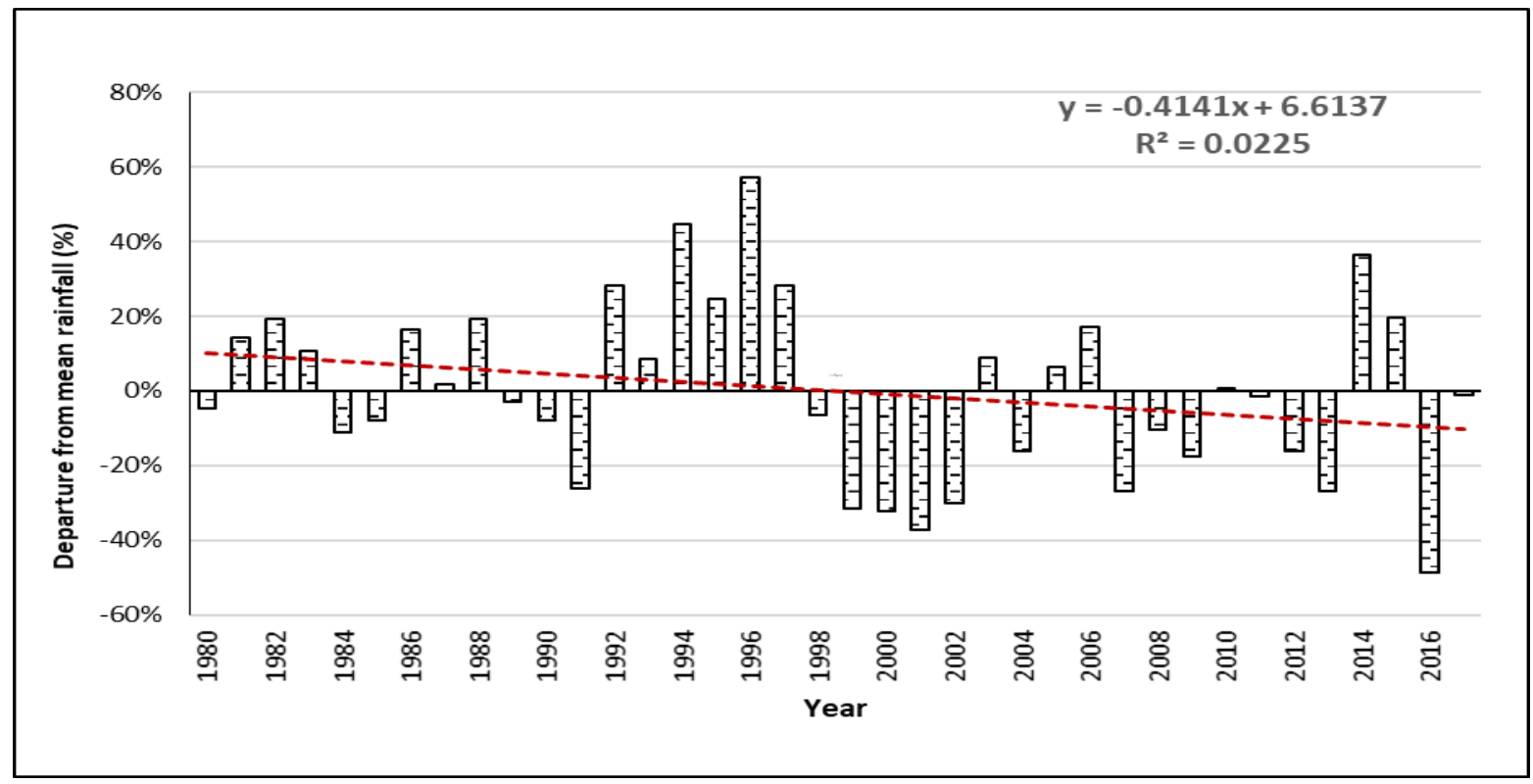


Fig.3 1-Month SPI for Anantnag district

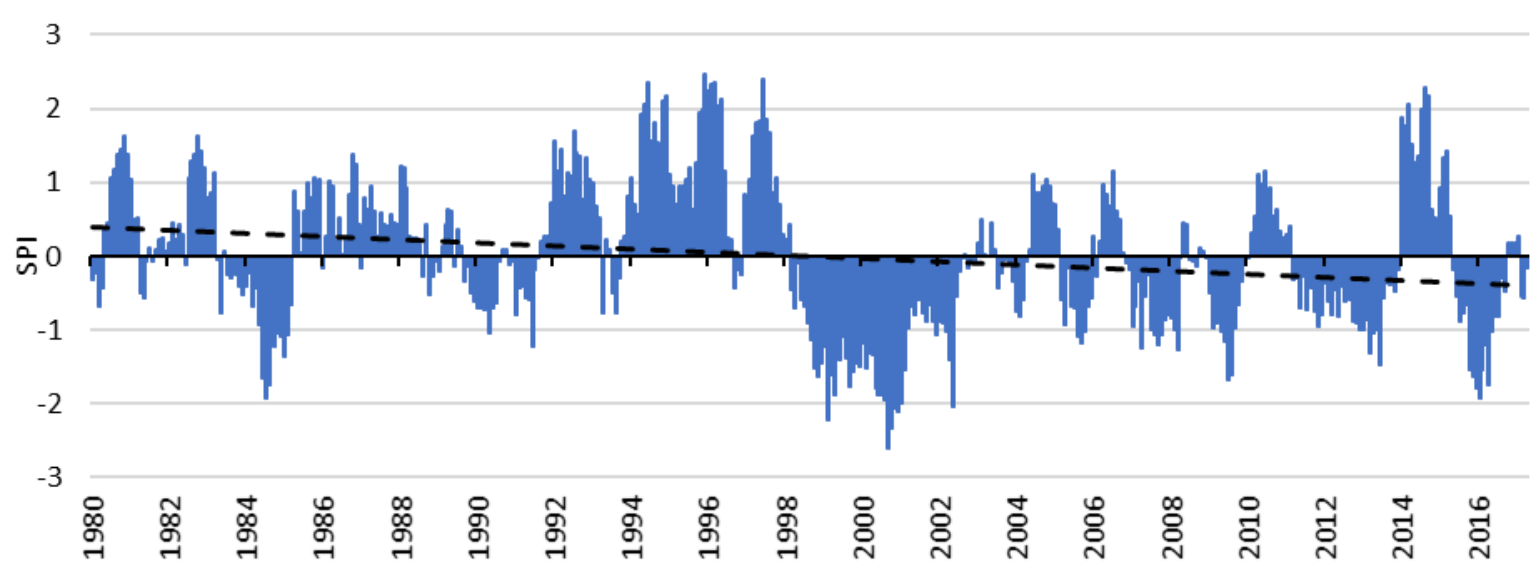

Fig.4 3-MONTH SPI FOR ANANTNAG DISTRICT

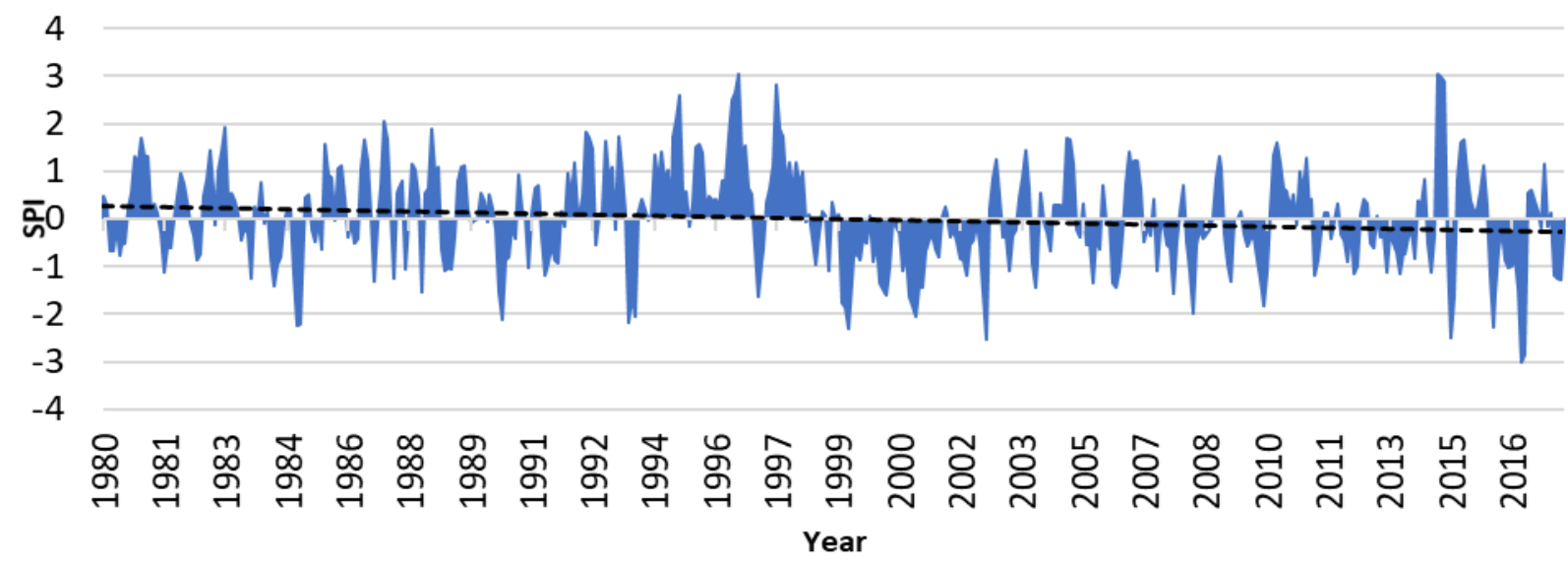

Fig.5 6-Month SPI for anantnag district

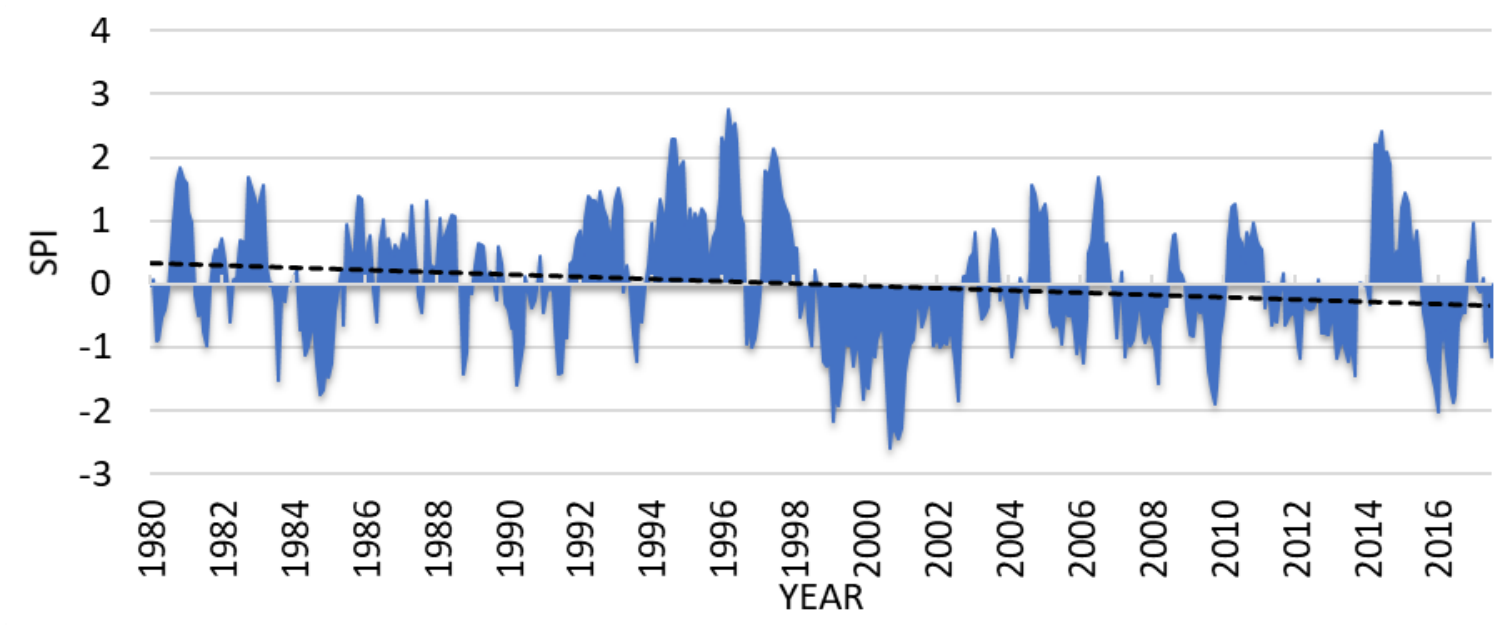


Fig.6 9-Month spi for anantnag district

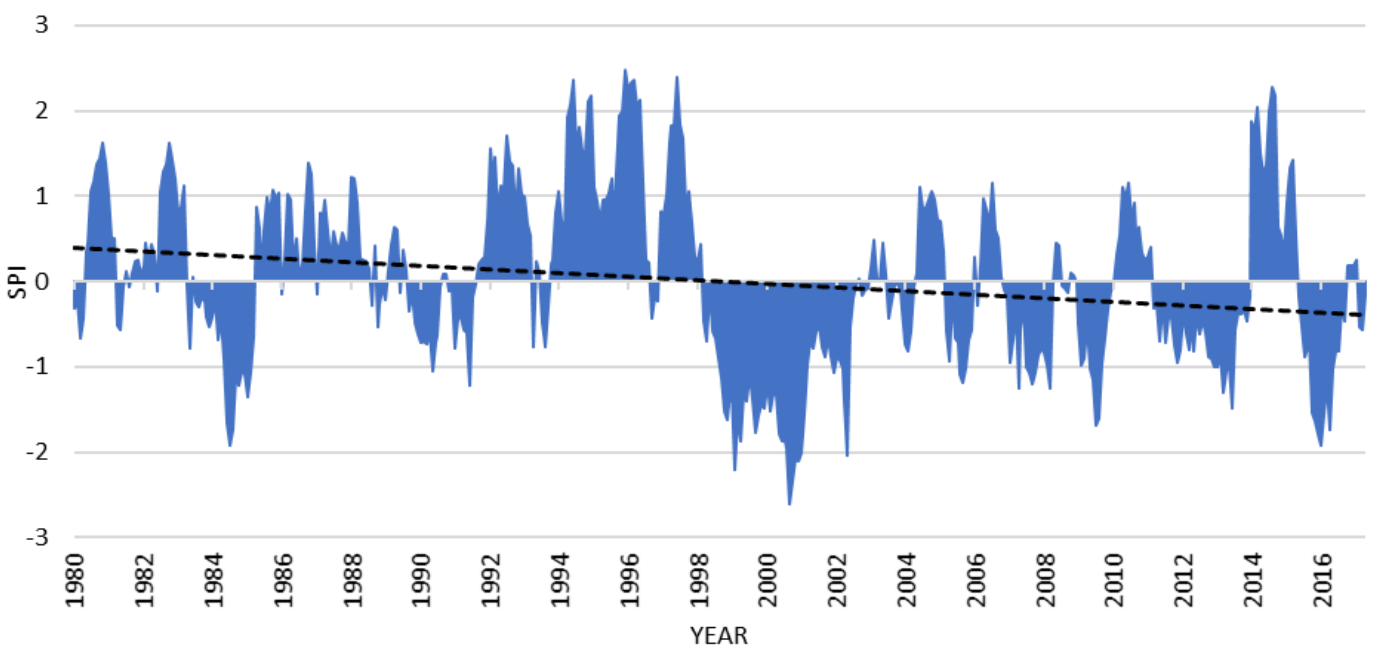

Fig. 7 12-Month spi for anantnag district

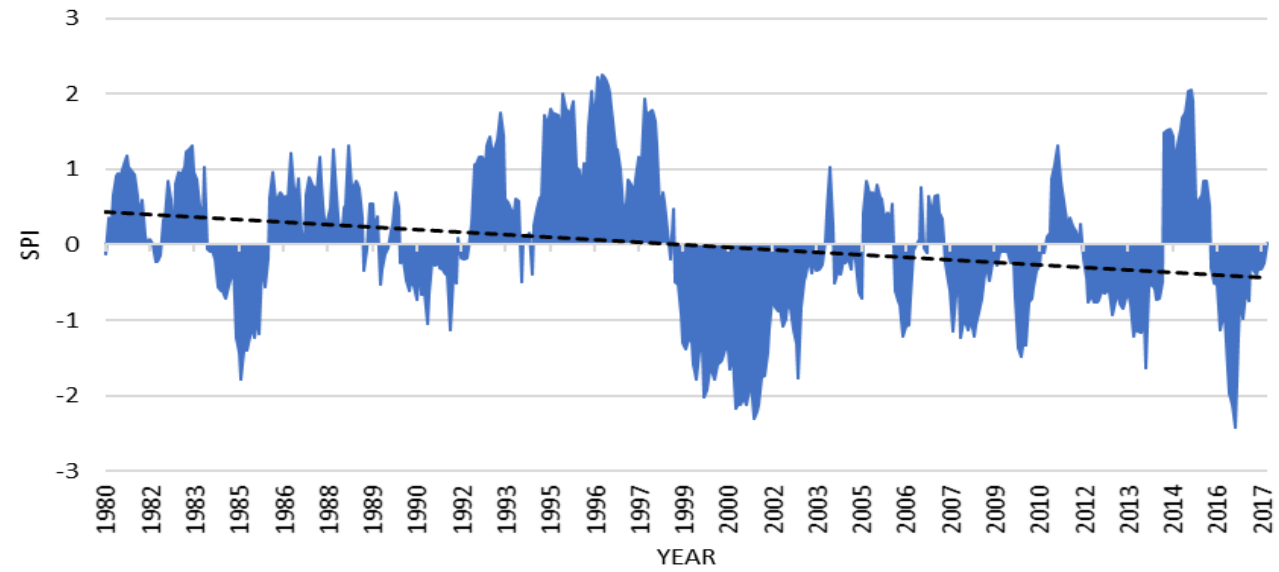

Fig. 8 The correlation between the percentage deviation from normal and the 12-Month SPI

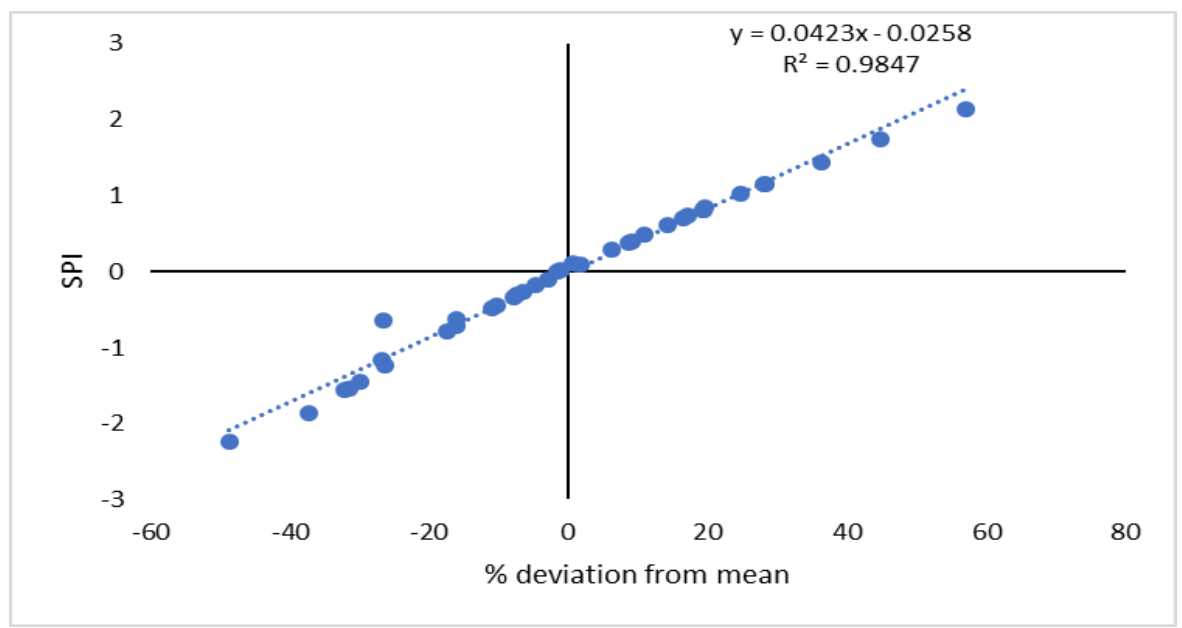




\section{2-Month SPI}

12- Month SPI values are generally linked to the stream flows, level of reservoirs and even groundwater levels at stretched time scales. The lowest SPI on 12- month time scale for Anantnag district is -2.42 which occurred during the year of 2016 (Fig. 7).

\section{Correlation between IMD criteria and SPI}

Correlation between the data obtained from IMD criteria and the SPI values of 12- month timescale was developed. The data of percentage deviation of precipitation from normal represents the data analysis based on IMD criteria. This data was correlated with the SPI values based on the temporal scale of 12-Month. From the study, it was observed that the data analysis obtained from the IMD criteria was $99 \%$ correlated with the results obtained from the SPI generator while taking temporal scale of 12-month SPI. The correlation between the percentage deviation from normal and the 12-Month SPI timescale is shown in the Fig. 8.

In conclusions, the main aim of this study was to assess droughts observed in the historical rainfall records of the Anantnag district by using the Standardized Precipitation Index criterion. Temporal drought patterns were examined which revealed many interesting results on the variability in the occurrence of drought in the region. A decreasing trend was observed in this district for the given rainfall data which indicates that the areas under consideration are drought prone. It was found that SPI is a valuable tool for assessing drought characteristics like frequency and severity.

The period of study during $1980-1997$ has not witnessed a single drought year. For the study period (last 40 years), only four Meteorological droughts occurred in the year of 1999, 2000, 2001 and 2016. All these droughts have occurred during the recent 20 years. Moreover, the SPI trend lines suggest decreasing nature of SPI on all scales. This implies that the frequency of droughts in the region has increased during last two decades. The decreasing trend of the precipitation further increases the vulnerability of the region to droughts. The worst period of the drought occurred during 1999 - 2001, where three consecutive drought years were observed in the region.

\section{References}

Ahmad, L., Parvaze, S., Majid, M. and Kanth, R.H., 2016. Analysis of Historical Rainfall Data for Drought Investigation Using Standard Precipitation Index (SPI) Under Temperate Conditions of Srinagar Kashmir. Pakistan Journal of Meteorology Vol, 13(25).

Bartolini E, Claps P, and D'Odorico P, 2008, Inter annual variability of winter precipitation in the European Alps: elations with the North Atlantic Oscillation, Hydrological Earth System Sciences. Discuss., 5, 2045-2065

Bordi I, Fraedrich K, Jiang M, and Sutera A, 2004, Spatio-temporal variability of dry and wet periods in eastern China, Theoretical Applied Climatology, 79, 81-91

Edwards D.C., McKee T.B., 1997, Characteristics of 20th century drought in the United States at multiple scales, Atmospheric Science, paper no. 634, May 1-30.

Giddings L., Soto M., Rutherford, B.M., and Maarouf, A., 2005, Standardized Precipitation Index zones for Mexico, Atmosphere, pp33-56.

Goodrich B Gregory and Ellis W Andrew, 2006, Climatological drought in Arizona: An analysis of indicators for guiding the Governor's drought task 
force, The Professional Geographer, 58, pp 460-469.

Guttman B Nathaniel, 1998, Comparing the

Palmer drought index and the standardized precipitation index, Journal of the American Water Resources Association, 34, 1, 113-121.

Guttman B Nathaniel, 1999, Accepting the Standardised Precipitation Index: A calculation algorithm, Journal of the American Water Resources Association, $35,2,311-322$.

Loukas A and Vasiliades L, 2004, Probabilistic analysis of drought spatiotemporal characteristics in Thessaly region, Greece, Natural Hazards and Earth System Sciences 4, 719-731

McKee B Thomas., Doesken J Nolan. and Kleist John, 1993, The relationship of drought frequency and duration to time scales, Proceedings of ninth Conference on Applied Climatology, American Meteorological Society, Boston, 179184.

McKee, T.B., Doesken, N.J., Kleist, J., 1995. Drought monitoring with multiple time scales. In: Proceedings of the 9th conference on Applied Climatology, AMS, Boston, MA, pp. 233-236.

Morid Saeid, Smakhtin Vladimir and Moghaddasi Mahnosh, 2006, Comparison of seven meteorological indices for drought monitoring in Iran, International Journal of Climatology, 26, 7, pp971-985

Parvaze, S., Parvaze, Saqib and Ahmad, L., 2018. Meteorological Drought Quantification with Standardized Precipitation Index for Jhelum Basin in Kashmir Valley. International Journal of Advance Research in Science and Engineering, 07, pp. 688-697.

Paulo A.A, Ferreira E, Coelho C and Pereira L.S, 2005, Drought class transition analysis through Markov and Loglinear models, an approach to early warning, Agricultural Water Management, Vol 77, Issues 1-3, Pages 59-81

Vicente-Serrano M Sergio, Gonzalez-Hidalgo Carlose Jose, Luis de Martin and Raventos Jose, 2004, Drought patterns in the Mediterranean area: the Valencia region (eastern Spain), Climate Research, 26, pp5-15.

Vijendra K Boken, 2005, Monitoring and Predicting Agricultural Drought - A Global Study, pp. 266-275, Oxford University Press.

Wu Hong, Svoboda D Mark, Hayes J. Michael, Wilhite A Donald and Fujiang Wen, 2006, Appropriate application of the standardizes precipitation index in arid locations and dry seasons, International Journal of Climatology, 27 , pp. 65-79.

\section{How to cite this article:}

Yogesh Pandey, Ram Nath Jha, Sabah Parvaze, Latief Ahmad and Shahzad Faisal. 2019. Standardized Precipitation Index (SPI) for Drought Intensity Assessment in South Kashmir. Int.J.Curr.Microbiol.App.Sci. 8(12): 2846-2856. doi: https://doi.org/10.20546/ijcmas.2019.812.332 